\title{
Large-amplitude longitudinal oscillations in solar prominences
}

\author{
Manuel Luna ${ }^{1,2}$, Judith Karpen ${ }^{3}$, Antonio Díaz ${ }^{1,2}$, Kalman \\ Knizhnik $^{4,3}$, Karin Muglach ${ }^{5,3}$, Holly Gilbert ${ }^{3}$, and Therese Kucera ${ }^{3}$ \\ ${ }^{1}$ Instituto de Astrofísica de Canarias, \\ E-38200 La Laguna, Tenerife, Spain \\ ${ }^{2}$ Universidad de La Laguna, Dept. Astrofísica, \\ E-38206 La Laguna, Tenerife, Spain \\ email: mluna@iac.es \\ ${ }^{3}$ NASA/GSFC, Greenbelt, MD 20771, USA \\ ${ }^{4}$ Johns Hopkins University, Baltimore, MD USA \\ ${ }^{5}$ ARTEP, Inc., Maryland, USA
}

\begin{abstract}
Large-amplitude longitudinal (LAL) prominence oscillations consist of periodic mass motions along a filament axis. The oscillations appear to be triggered by an energetic event, such as a microflare, subflare, or small C-class flare, close to one end of the filament. Observations reveal speeds of several tens to $100 \mathrm{~km} / \mathrm{s}$, periods of order $1 \mathrm{hr}$, damping times of a few periods, and displacements equal to a significant fraction of the prominence length. We have developed a theoretical model to explain the restoring force and the damping mechanism. Our model demonstrates that the main restoring force is the projected gravity in the flux tube dips where the threads oscillate. Although the period is independent of the tube length and the constantly growing mass, the motions are strongly damped by the steady accretion of mass onto the threads. We conclude that the LAL movements represent a collective oscillation of a large number of cool, dense threads moving along dipped flux tubes, triggered by a nearby energetic event. Our model yields a powerful seismological method for constraining the coronal magnetic field strength and radius of curvature at the thread locations.
\end{abstract}

Keywords. Solar prominence, Oscillations, Magnetic structure.

\section{Observational LAL Oscillations Features}

Large-amplitude longitudinal (LAL) oscillations in prominences were first reported by Jing et al. (2003); few additional observations have been reported which are summarized in Table 1 (Jing et al. 2006, Vršnak et al. 2007, and Zhang et al. 2012). The oscillations are longitudinal because the displacements are mainly along the axis of the filament or slightly skewed. According to the standard filament models and sparse observations of filament magnetic fields (see, Trujillo-Bueno et al. 2002 \& Casini et al. 2003) the LAL displacements are along the field. These oscillations have long periods of 50-160 minutes, and are damped in 2.3-6.2 cycles, with high velocity amplitudes in the range $30-100 \mathrm{~km} \mathrm{~s}^{-1}$. LAL oscillations are apparently triggered by an energetic event: a subflare, a microflare, or a flare close to the filament in all reported events.

\section{Theoretical Interpretation}

LAL oscillation events are impressive due to the high speeds and large displacements reached by the massive prominences. Although the energy and momentum of the prominence are huge, the acceleration reached in the oscillatory motion can be as large as 
Table 1. Some observational features of LAL oscillations reported so far.

\begin{tabular}{|c|c|c|c|c|}
\hline | Observation & Amp. $\left(k m s^{-1}\right)$ & Period $(\min )$ & $\mid$ Damping Time (min) $\mid$ & Trigger \\
\hline Jing et al. (2003) & 92 & 80 & $210(2.6$ periods $)$ & | Nearby sub-flare \\
\hline | Jing et al. (2006) & 50 & 160 & $600(3.8$ periods $)$ & | Nearby C-Class flare \\
\hline | Jing et al. (2006) & 30 & 150 & - & | Nearby microflare \\
\hline | Jing et al. (2006) & 100 & 100 & - & - \\
\hline |Vršnak et al. (2007)| & 51 & 50 & 115 (2.3 periods $)$ & | Nearby sub-flare \\
\hline | Zhang et al. (2012) | & 40 & 52 & 133 (2.6 periods) & | Nearby C-Class flare \\
\hline |Li \& Zhang (2012) & $30-60$ & $44-67$ & - & | Nearby flare activity \\
\hline
\end{tabular}

$100 \mathrm{~m} \mathrm{~s}^{-2}$, comparable to the solar gravity acceleration $\left(g_{0}=274 \mathrm{~m} \mathrm{~s}^{-2}\right)$. This motion is rapidly damped in only a few periods, demonstrating that a very efficient damping mechanism is operating. Several mechanisms have been proposed to explain the restoring force and damping of the LAL oscillations, but most of them do not successfully describe the thread motions (see review by Tripathi et al. 2009). Some authors claim that the restoring force is associated with the magnetic tension; in that case, the associated displacements would be perpendicular to the local magnetic field, contrary to what the observations show. Another possible restoring force is the gas pressure (slow MHD mode), but the temperatures necessary to accelerate the prominence threads to observed values are on the order of $10 \mathrm{MK}$. Although these temperatures are not observed in the oscillations, they could be present in the initiation event, e.g. a flare.

We have studied the oscillations of threads forming the basic components of a prominence (Luna \& Karpen 2012) in a 3D sheared arcade (DeVore et al. 2005, Luna et al. 2012a). According to the thermal nonequilibrium model (Antiochos et al. 1999), the cool prominence threads form as the result of heating at the base of long coronal loops in filament channels, which produces chromospheric evaporation and subsequent dynamic radiative collapse. Inspired by the transient oscillations seen in our earlier simulations of this process, we developed the first self-consistent model for the observed large-amplitude oscillations along filament axes that explains both the restoring force and the damping mechanism. Because the corona is low- $\beta$, it is reasonable to assume that the threads are ducted along concave-upward (dipped) magnetic flux tubes as a solid body. We also assume that the density of the threads is much larger than the ambient coronal density. The simulations reveal that the pressure difference force between both ends of the thread is too small to produce the acceleration associated with the oscillations (see details in Luna et al. 2012a). Thus, the pressure force is not the restoring mechanism in the LAL oscillations, and the corona has little influence on the thread dynamics.

As long as the footpoint heating is steady, the mass of the threads continuously increases with time $(t)$ by accretion of evaporated chromospheric plasma. Our simulations show that $m(t)=m_{0}+\alpha\left(t-t_{0}\right)$ with a typical accretion rate $\alpha=20 \times 10^{6} \mathrm{~kg} / \mathrm{hr}$. The center of mass position of each thread, $s$, is described by a zero-order Bessel equation (see Eq. 2 in Luna \& Karpen 2012). The solutions are the zero-order Bessel functions that are intrinsically damped. However, it is necessary to introduce an additional damping term, $e^{-\left(t-t_{0}\right) / \tau_{\mathrm{w}}}$, in order to account for additional weak damping found in the simulations. With these considerations the motion of the threads is described by

$$
s(t)=s_{0}+A J_{0}\left[\omega\left(t-t_{0}\right)+\omega m_{0} / \alpha\right] e^{-\left(t-t_{0}\right) / \tau_{\mathrm{w}}} .
$$


The angular frequency of the oscillations is

$$
\omega=\sqrt{\frac{g_{0}}{R}},
$$

where $R$ is the radius of curvature of the dipped portion of the flux tubes supporting the threads. This oscillation resembles the motion of a gravity-driven pendulum. In Luna et al. (2012b) we studied the normal modes of oscillation of threads in curved field lines, and found that there are two contributions to the oscillation frequency: the gravity-driven term (Eq. 2.2) and the pressure-driven slow mode. For typical prominence parameters the pressure-driven term is small, however, confirming that the restoring force is the projected gravity. Equation (2.2) allows us to directly calculate the radius of curvature of the field lines that support the prominence.

Two mechanisms damp the thread motion (Eq. 2.1). The most important is associated with the mass accretion and related to the phase of the Bessel function $\omega m_{0} / \alpha$. For a large accretion rate $\alpha$, the phase is close to zero and the Bessel function is strongly damped. In contrast, for small values of $\alpha$ the phase is large and the Bessel function is essentially undamped. The second mechanism is the weak damping characterized by $\tau_{\mathrm{w}}$. The simulations show that this is unimportant during the first oscillations of the threads. We speculate that the weak damping is associated with a non-adiabatic effect of energy dissipation.

The observed LAL oscillations appear to be triggered by small flares close to the filaments. However, the precise mechanism is not known. In Luna \& Karpen (2012) we proposed that the energetic event increases the heating impulsively at one end of the filament, increasing the evaporation of chromospheric plasma into the affected flux tubes. We speculate that extra heat is deposited only on those footpoints located closest to the energetic event, so only parts of the filament are excited simultaneously and oscillate collectively in phase. Zhang et al. (2013) studied numerically the triggering by impulsive microflare heating of one leg of a prominence, and found that threads start to oscillate. This study revealed that condensations are remarkably robust; increased heating at one footpoint produces a flow of hot evaporated plasma that pushes a thread to a new location, rather than destroying it in situ. Note that this result is consistent with our earlier studies of thermal nonequilibrium (e.g., Karpen \& Antiochos 2008).

\section{Prominence Seismology}

It is generally agreed that the dense prominence threads must be magnetically supported. Thus, the magnetic tension in the dipped part of the tubes must be larger than the weight of the threads. With this consideration and Equation (2.2), we derived the following equation that constrains the minimum field strength as a function of the thread oscillation period (see Eq. 7 of Luna \& Karpen 2012):

$$
B[\mathrm{G}] \geqslant 26\left(\frac{n}{10^{11} \mathrm{~cm}^{-3}}\right)^{1 / 2} P[\text { hours }] .
$$

For typical electron densities $\left(n \sim 10^{11} \mathrm{~cm}^{-3}\right)$ and LAL oscillation periods $(\sim 1$ hour $)$ the estimated magnetic field is consistent with the sparse observations of intermediate-type prominence magnetic fields of around 20 Gauss (see review by Mackay et al. 2010 \& T. Berger paper in this volume).

In addition to providing valuable information on the difficult-to-measure coronal magnetic field in filament channels, our model can also determine the radius of curvature of the prominence flux-tube dips from the observed oscillation properties. We have used the 
Jing et al. (2003) and Vršnak et al. (2007) observations to solve Equation (2.2) for $R$. The observed periods are $80 \mathrm{~min}$ and $50 \mathrm{~min}$ respectively, yielding corresponding curvature radii of $152 \mathrm{Mm}$ and $62 \mathrm{Mm}$. Assuming a typical prominence density of $n=10^{11} \mathrm{~cm}^{-3}$, we estimate the minimum field strength in the two prominences as $\sim 35 \mathrm{G}$ and $22 \mathrm{G}$.

The capabilities of recent instruments such as AIA/SDO (see Lemen et al. 2012) and NASA's Interface Region Imaging Spectrograph (IRIS) allow more LAL events to be discovered and characterized. We have identified several LAL events in the AIA data, and have analysed the oscillations of one such filament observed on August 20, 2010. The analysis and results will be described in a forthcoming publication (Luna et al. 2013); preliminary results are presented in the Knizhnik et al. paper in this volume.

\section{Conclusions}

We conclude that the observed LAL motions represent a collective oscillation of many cool, dense threads moving along the magnetic field, triggered by a nearby energy release event (e.g. a small flare). The main restoring force is the projected gravity in the flux tube dips where the threads oscillate, even in threads with large radii of curvature. The period is independent of the tube length and of the constantly growing mass, but the oscillations are strongly damped by the mass accretion caused by persistent footpoint heating. After several oscillations the excited threads lose their coherence due to slight differences in the oscillation periods, causing different parts of the filament to oscillate increasingly out of phase as observed. The mechanism that initiates the collective oscillation is not determined in the present investigation. We speculate that the impulsive energetic event temporarily increases the evaporation at the closest filament flux-tube footpoints. This raises the plasma pressure only at one end of the threads, initiating the oscillations. More observations of oscillating prominences are needed to further refine and test our model.

\section{References}

Antiochos, S. K., MacNeice, P. J., Spicer, D. S., \& Klimchuk, J. A. 1999, ApJ, 512, 985

Casini, R., López Ariste, A., Tomczyk, S., \& Lites, B. W. 2003, ApJL, 598, L67

DeVore, C. R., Antiochos, S. K., \& Aulanier, G. 2005, ApJ, 629, 1122

Jing, J., Lee, J., Spirock, T. J., et al. 2003, ApJL, 584, L103

Jing, J., Lee, J., Spirock, T. J., \& Wang, H. 2006, Sol. Phys., 236, 97

Karpen, J. T. \& Antiochos, S. K. 2008, ApJ, 676, 658

Lemen, J. R., Title, A. M., Akin, D. J., et al. 2012, Sol. Phys., 275, 17

Luna, M., Karpen, J. T., \& Devore, C. R. 2012a, ApJ, 746, 30

Luna, M. \& Karpen, J. 2012, ApJ, 750, L1

Luna, M., Díaz, A. J., \& Karpen, J. 2012b, ApJL, 757, L98

Luna, M., Knizhnik, K., Muglach, K., Gilbert, H, Kucera, T., \& Karpen, 2013, in preparation

Mackay, D. H., Karpen, J. T., Ballester, J. L., Schmieder, B., \& Aulanier, G. 2010, Space Sci. Rev., 151, 333

Tripathi, D., Isobe, H., \& Jain, R. 2009, Space Sci. Rev., 149, 283

Trujillo Bueno, J., Landi Degl'Innocenti, E., Collados, M., Merenda, L., \& Manso Sainz, R. 2002, Nature, 415, 403

Vršnak, B., Veronig, A. M., Thalmann, J. K., \& Žic, T. 2007, Astron. Astrophys., 471, 295

Zhang, Q. M., Chen, P. F., Xia, C., \& Keppens, R. 2012, Astron. Astrophy., 542, A52

Zhang, Q. M., Chen, P. F., Xia, C., Keppens, R., \& Ji, H. S. 2013, Astron. Astrophys., 554, A124 